\title{
Topological design considering flexibility under periodic loads
}

\author{
S. Nishiwaki, K. Saitou, S. Min, and N. Kikuchi
}

\begin{abstract}
Topology optimization has been extensively considered to design the structural configuration for the stiffness maximization and the eigenfrequency maximization. In this paper, we construct a topology optimization method implementing flexibility with the time-periodic loading condition. First, the flexibility in the dynamic periodic loading is formulated using the mutual energy concept. Second, the multi-optimization problem is formulated using a new multi-objective function in order to obtain an optimal solution incorporating both flexibility and stiffness. Next, the topology optimization procedure is developed using the homogenization design method. Finally, some examples are provided to confirm the optimal design method presented here.
\end{abstract}

\section{1}

\section{Introduction}

We shall discuss a structural optimization method which implements structural flexibility in the case where structures are subjected to a periodic applied force in time using the homogenization design method. In general structural design, the stiffest structure has been considered optimal. However, by implementing flexibility in appropriate portions of the structure, we can obtain additional functions such as the mechanical function. A typical example of a structure with an additional mechanical function is a compliant mechanism (a flexible structure). It is designed to be flexible in order to achieve a specified motion.

The idea of incorporating fiexibility into a structure is not new. The earliest effort was made by Burns and

Received January 18, 1999

S. Nishiwaki ${ }^{1}$, K. Saitou ${ }^{2}$, S. Min $^{3}$, and N. Kikuchi ${ }^{4}$

${ }^{1,2,4}$ Department of Mechanical Engineering and Applied Mechanics, The University of Michigan, Ann Arbor, MI, 48109-2125, USA

${ }^{3}$ School of Mechanical Engineering, Hanyang University, 17

Haengdang-Dong Sungdong-ku, Seoul, 133-791 Korea
Crossley (1966). Later, Midha and his associates developed a design method based on traditional rigid body kinematics (e.g. Her and Midha 1987). On the other hand, Ananthasuresh et al. (1994), Sigmund (1996), and Nishiwaki et al. (1998) constructed design methods based on the topology optimization technique. However, these works focus on the design of compliant mechanisms for static force input and do not consider the situation where compliant mechanisms are subjected to periodic loads in time.

It is also noted that we can design a high frequency actuator or a mechanical transducer by combining a flexible structure with excitation devices such as piezo-ceramic or electromagnetic devices (e.g. Onituska et al. 1995). Since these devices have small time constants, they can provide high frequency excitation. One drawback of such devices is that the output displacement is generally too small for use in an actuator or mechanical transducer. However, combining these devices with flexible structures can overcome this problem. That is, the mechanical structure can amplify the device displacement using flexibility. Typical applications are the design of the ultrasonic motor and the ultrasonic travelling wave linear motor (Seemann 1996; Zhang and Zhu 1997). While several methods have been presented for analysis of this type of high frequency actuator using FEM (see e.g. Kagawa et al. 1996), a design method of a flexible structure for high frequency actuators has not been established.

Viewed as resonators, flexible structures under periodic loads have been of great interest in the field of micro-electro-mechanical systems (MEMS) where electrical circuitry and mechanical structures are integrated in micrometer scale by using IC fabrication processes. Micro mechanical resonators, typically implemented as micro cantilever or fixed-supported beams, are central components of many MEMS devices including sensors, filters and oscillators, and actuators, which realize significant size reduction while achieving narrow bandwidth (i.e. high $Q$ factor) and improved frequency stability (Howe 1994). Micro mechanical resonators have been successfully applied to sensors for linear acceleration (Allen et al. 1989), angular rotation rate (Greiff et al. 1991) and pressure (Thornton et al. 1988). Micro mechanical resonators have also been used as an alternative to off chip 
quartz crystal and SAW resonators for wireless communication (Nguyen 1996). Other application areas include torsional scanning mirrors (Petersen 1980), ultrasound sources (Huang and Kuratli 1996), and microvibromotors that translate mechanical vibration to rotary (Lee and Pisano 1992) or linear motion (Daneman et al. 1996; Saitou and Wou 1998). Despite such wide application areas, all of these works use essentially the same resonator design and do not discuss general methods for synthesizing the optimal resonator structures.

The homogenization design method has been widely used for structural topology design since Bendsøe and Kikuchi (1988) proposed it. The key ideas of the method are (1) the extension of a design domain to a fixed domain using the characteristic function which Murat and Tartar (1985) proposed, and (2) utilization of the homogenization method to deal with wild discontinuity of the extended design domain. Thus, the structural optimization problem is replaced with a problem of distributing the porous media in the fixed domain. This method has been extended to a variety of structural optimization problems such as the minimum compliance problem (Suzuki and Kikuchi 1991) and the eigenfrequency problem (Ma et al. 1995).

In this paper, we shall develop a methodology in which the homogenization design method is used to obtain the optimal structure design considering flexibility for cases in which the boundary is under a periodic load in time. In Section 2, the homogenization design method is briefly discussed. In Section 3, the flexibility and stiffness under periodic loads in time are formulated using the mutual energy concept. Their sensitivities with respect to a design variable are also derived. In Section 4 , the multioptimization problem is formulated in order to design a flexible structure under a periodic load in time. A new multi-objective function is also formulated in order to obtain appropriate optimal solutions in the Pareto optima. In Section 5, the optimization algorithm is constructed using the homogenization method and sequential linear programming (SLP). Finally, in Section 6, some design examples are presented in order to examine the optimal configurations. These examples confirm that the methodology presented here can provide flexible structures for application to the design of high frequency actuators and resonators.

\section{2}

\section{Homogenization design method}

The homogenization design method, first constructed by Bendsøe and Kikuchi (1988), is briefly discussed in this section. Consider the design problem determining the boundary of the design domain $\Omega_{d}$ by minimizing or maximizing objective functions. The key idea of this method is the introduction of a fixed and extended design domain $D$ including the original design domain $\Omega_{d}$, a priori, and the utilization of the following character- istic function introduced by Murat and Tartar (1985) to describe any boundaries of the original design domain $\Omega_{d}$ :

$\chi_{\Omega}(\mathbf{x})=\left\{\begin{array}{l}1 \text { if } \mathbf{x} \in \Omega_{d}, \\ 0 \text { if } \mathbf{x} \in D \backslash \Omega_{d} .\end{array}\right.$

Using this function, the original structural design problem is replaced with the material distribution problem of the new elasticity tensor, $\chi_{\Omega} \mathbf{E}$, and the new mass density, $\chi_{\Omega} \varrho$, in the extended design domain $D$, where $\mathbf{E}$ is the elasticity tensor and $\varrho$ is the mass density in the original design $\Omega_{d}$. However, this characteristic function is very discontinuous everywhere. This nature makes numerical treatment impossible. To overcome this problem, the homogenization method was employed (Bendsøe and Kikuchi 1988; Kohn and Strang 1986; Murat and Tartar 1985). In this method, wildly discontinuous properties are replaced with continuous and equivalent ones in the global sense.

Next, the procedure for the homogenization method is briefly explained. Figure 1 shows a microstructure used for the relaxation of the design domain in the two-dimensional problem. The microstructure is formed inside an empty rectangle in a unit cell, where $\alpha, \beta$, and $\theta$ are regarded as the design variables. In order to develop a complete void, both $\alpha$ and $\beta$ must be 1 , whereas for solid material, $\alpha$ and $\beta$ must be 0 . The variable $\theta$ represents the rotation of the unit cell. Note that the optimal solutions obtained by the rectangle type microstructure are sub-optimal. We must use the rank- $n$ type microstructure (e.g. see Bendsøe 1995, pp. 9-19) to obtain the true optimal solutions. However, the rank- $n$ type microstructure provides many gray scale regions implying a composite status in the optimal configurations. Several methods, such as the perimeter method (Haber et al. 1996) are proposed to eliminate gray scale regions from the optimal configurations. However, these methods do not work well for all design problems (Fujii and Kikuchi 1998). On the other hand, the rectangle type microstructure provides a clear and appropriate optimal configuration which does not have many gray regions in the engineering sense. This is because the rectangle type microstructure has a penalty characteristic between the elasticity tensor and the density of the microstructure. Thus, we employ the rectangle type microstructure in this research.

The elasticity tensor $\chi_{\Omega} \mathbf{E}$ and the mass density $\chi_{\Omega} \varrho$ are rewritten as $\mathbf{E}^{\varepsilon}$ and $\varrho^{\varepsilon}$, respectively. Suppose that elasticity tensor $\mathbf{E}^{\varepsilon}$ has the $Y$-periodic characteristic in order to occupy some portions in the extended design domain $D$ with the infinite microstructures shown in Fig. 1. Using parameter $\varepsilon$, which represents the periodicity and is assumed to be very small, the local coordinate $\mathbf{y}$ in the microstructure is defined by

$\mathbf{y}=\mathbf{x} / \varepsilon$ 


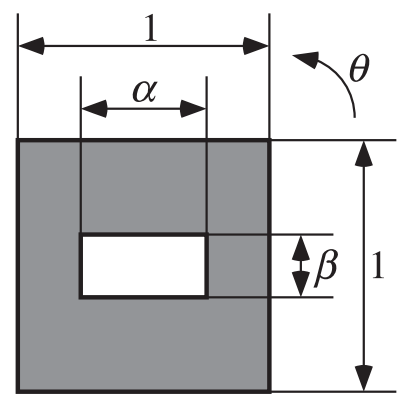

Fig. 1 A microstructure for the relaxation of the design domain

Using this local coordinate, elasticity tensor $\mathbf{E}^{\varepsilon}$ and mass density $\varrho^{\varepsilon}$ are described as

$\mathbf{E}^{\varepsilon}(\mathbf{x})=\mathbf{E}(\mathbf{x}, \mathbf{y}), \quad \varrho^{\varepsilon}(\mathbf{x})=\varrho(\mathbf{x}, \mathbf{y})$.

First, homogenized elasticity tensor $\mathbf{E}^{H}$ and homogenized mass density $\varrho^{H}$ in the case where the angle $\theta$ is set to 0 are calculated. To obtain this homogenized elasticity tensor, characteristic deformations $\boldsymbol{\chi}(\mathbf{x}, \mathbf{y})$ are calculated using the following equation:

$$
\begin{aligned}
& \int_{\mathbf{Y}} \varepsilon_{\mathbf{y}}(\mathbf{v})^{T} \mathbf{E}(\mathbf{x}, \mathbf{y}) \varepsilon_{\mathbf{y}}[\chi(\mathbf{x}, \mathbf{y})] \mathrm{d} y= \\
& \int_{\mathbf{Y}} \mathbf{v} \varepsilon_{\mathbf{y}}(\mathbf{v})^{T} \mathbf{E}(\mathbf{x}, \mathbf{y}) \mathrm{d} Y, \quad \text { for } \forall \mathbf{v} \in V_{y},
\end{aligned}
$$

where $\boldsymbol{\varepsilon}_{\mathbf{y}}()^{T}=\left\{\frac{\partial}{\partial y_{1}} \frac{\partial}{\partial y_{2}} \frac{1}{2}\left(\frac{\partial}{\partial y_{2}}+\frac{\partial}{\partial y_{1}}\right)\right\}$, and $V_{y}$ is the admissible space defined in the unit cell $Y$ such that $V_{y}=$ $\left\{\mathbf{v}=v_{i} \mathbf{e}_{i}: v_{i} \in H^{1}(\mathbf{Y}) \mid \mathbf{v}\right.$ is $Y$-periodic in the unit cell $\left.Y\right\}$. After obtaining characteristic deformations $\chi(\mathbf{x}, \mathbf{y})$, homogenized elasticity tensor $\mathbf{E}^{H}$ and homogenized mass density $\varrho^{H}$ are computed by

$\mathbf{E}^{H}=\frac{1}{|Y|} \int_{\mathbf{Y}} \mathbf{E}(\mathbf{x}, \mathbf{y})\left[\mathbf{I}-\boldsymbol{\varepsilon}_{\mathbf{y}}(\boldsymbol{\chi})\right] \mathrm{d} Y$,

$\varrho^{H}=\frac{1}{|Y|} \int_{\mathbf{Y}} \varrho(\mathbf{x}, \mathbf{y}) \mathrm{d} Y$,

where $|Y|$ stands for the area of the unit cell.

Next, when the unit cell is rotated by angle $\theta$, homogenized elasticity tensor $\mathbf{E}^{G}$ is computed by

$\mathbf{E}^{G}=\mathbf{R}(\theta)^{T} \mathbf{E}^{H} \mathbf{R}(\theta)$,

where $\mathbf{R}$ is the rotation matrix. Thus, the homogenized properties are determined by the microscopic design variables $\alpha, \beta$, and $\theta$.

\section{Formulation of flexibility and stiffness under periodic loads}

In this section, we formulate the flexibility and stiffness in the case where the periodic loads in time are applied using the mutual energy concept introduced by Shield and Prager (1970) and Huang (1971). Suppose that an elastic body occupying a two-dimensional domain, $\Omega$, is fixed at boundary $\Gamma_{d}$. Now we consider the two equilibrium cases with a different traction in a different boundary: Case (a) and Case (b), as shown in Fig. 2. That is, in Case (a), the elastic body is subjected to boundary traction $\mathbf{t}^{1}$ at boundary $\Gamma_{t^{1}}$, and in Case (b), it is subjected to boundary traction $\mathbf{t}^{2}$ at boundary $\Gamma_{t^{2}}$. Body forces applied to the elastic body and the damping effect are assumed to be ignored for simplicity in the formulation. The displacement fields are $\mathbf{u}^{1}=\left\{u_{1}^{1}, u_{2}^{1}\right\}^{T}$ in Case (a), and $\mathbf{u}^{2}=\left\{u_{1}^{2}, u_{2}^{2}\right\}^{T}$ in Case (b).

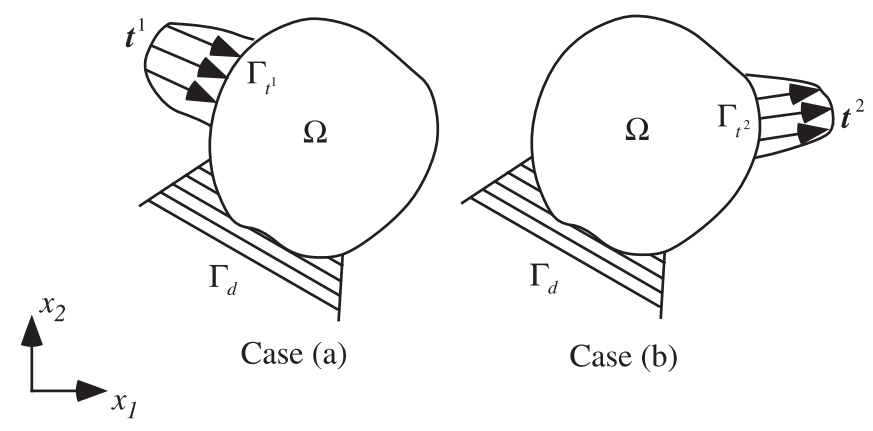

Fig. 2 An elastic body subjected to two tractions

We suppose that tractions $\mathbf{t}^{1}$ and $\mathbf{t}^{2}$ are harmonic excitations to the elastic body, and displacement fields $\mathbf{u}^{1}$ and $\mathbf{u}^{2}$ are also harmonic in the steady state. That is, tractions $\mathbf{t}^{1}$ and $\mathbf{t}^{2}$ and displacement fields $\mathbf{u}^{1}$ and $\mathbf{u}^{2}$ are assumed to be described as $\mathbf{t}^{1}=\mathbf{T}^{1} e^{j \omega t}, \mathbf{t}^{2}=\mathbf{T}^{2} e^{j \omega t}$, $\mathbf{u}^{1}=\mathbf{U}^{1} e^{j \omega t}$, and $\mathbf{u}^{2}=\mathbf{U}^{2} e^{j \omega t}$, where $\omega$ stands for an excitation frequency, $t$ stands for time, and $\mathbf{T}^{1}, \mathbf{T}^{2}, \mathbf{U}^{1}$, and $\mathbf{U}^{2}$ stand for amplitudes of $\mathbf{t}^{1}, \mathbf{t}^{2}, \mathbf{u}^{1}$, and $\mathbf{u}^{2}$, respectively.

Here, we introduce the linear form implying the mutual mean compliance in the dynamic problem defined by

$L^{2}\left(\mathbf{U}^{1}\right)=\int_{\Gamma_{t^{2}}} \mathbf{T}^{2} \bullet \mathbf{U}^{1} \mathrm{~d} \Gamma=\int_{\Gamma_{t^{2}}} T_{i}^{2} U_{i}^{1} \mathrm{~d} \Gamma, \quad \mathbf{U}^{1} \in V$,

where $V$ is the admissible linear space such that $V=$ $\left\{\mathbf{v}=v_{i} \mathbf{e}_{i}: v_{i} \in H^{1}(\Omega)\right.$ with $\mathbf{v}=0$ on $\left.\Gamma_{d}, i=1,2\right\}$. We assume that boundary traction $\mathbf{t}^{1}$ in Case (a) is an applied force, and the amplitude of boundary traction $\mathbf{T}^{2}$ 
in Case (b) is a unit dummy vector. Then, mutual mean compliance $L^{2}\left(\mathbf{U}^{1}\right)$ represents the measure of deformation at boundary $\Gamma_{t^{2}}$ when we apply boundary traction $\mathbf{t}^{1}$ at boundary $\Gamma_{t^{1}}$. That is, mutual mean compliance $L^{2}\left(\mathbf{U}^{1}\right)$ is interpreted as how flexible or stiff boundary $\Gamma_{t^{2}}$ is when boundary traction $\mathbf{t}^{1}$ is applied at boundary $\Gamma_{t^{1}}$ in the dynamic case. Therefore, by maximizing or increasing the absolute value of $L^{2}\left(\mathbf{U}^{1}\right)$, we can obtain sufficient flexibility along a direction specified by $\mathbf{T}^{2}$ with respect to $\mathbf{t}^{1}$. Note that both the maximization of $L^{2}\left(\mathbf{U}^{1}\right)$ and minimization of $L^{2}\left(\mathbf{U}^{1}\right)$ provide sufficient flexibility, because under harmonic excitation, the deformation in the direction of $-\mathbf{T}^{2}$, where the phase angle is $\omega t$, is identical to the deformation in the direction of $\mathbf{T}^{2}$, where the phase angle is $\omega t+\pi$.

We also introduce the following bilinear forms to describe the equilibrium equations in the weak form:

$$
\begin{aligned}
& a(\mathbf{u}, \mathbf{v})=\int_{\Omega} \varepsilon(\mathbf{v})^{T} \mathbf{E} \boldsymbol{\varepsilon}(\mathbf{u}) \mathrm{d} \Omega= \\
& \int_{\Omega} E_{i j k \ell} \varepsilon_{k \ell}(\mathbf{u}) \varepsilon_{i j}(\mathbf{v}) \mathrm{d} \Omega,
\end{aligned}
$$

with linearized strains

$\varepsilon(\mathbf{u})=\varepsilon_{i j}(\mathbf{u})=\frac{1}{2}\left(\frac{\partial u_{i}}{\partial x_{j}}+\frac{\partial u_{j}}{\partial x_{i}}\right)$,

and

$b(\mathbf{u}, \mathbf{v})=\int_{\Omega} \varrho \mathbf{u} \bullet \mathbf{v} \mathrm{d} \Omega=\int_{\Omega} \varrho \mathbf{u}^{T} \mathbf{v} \mathrm{d} \Omega$,

where $E_{i j k \ell}$ is the elasticity tensor and $\varrho$ is the mass density.

Since the displacement fields satisfy, respectively, the following equilibrium equations:

$a\left(\mathbf{U}^{1}, \mathbf{v}^{1}\right)-\omega^{2} b\left(\mathbf{U}^{1}, \mathbf{v}^{1}\right)=L^{1}\left(\mathbf{v}^{1}\right)$,

for $\mathbf{U}^{1} \in V, \quad \forall \mathbf{v}^{1} \in V$,

$a\left(\mathbf{U}^{2}, \mathbf{v}^{2}\right)-\omega^{2} b\left(\mathbf{U}^{2}, \mathbf{v}^{2}\right)=L^{2}\left(\mathbf{v}^{2}\right)$,

for $\mathbf{U}^{2} \in V, \quad \forall \mathbf{v}^{2} \in V$,

the displacement fields also satisfy, respectively,

$a\left(\mathbf{U}^{1}, \mathbf{v}^{2}\right)-\omega^{2} b\left(\mathbf{U}^{1}, \mathbf{v}^{2}\right)=L^{1}\left(\mathbf{v}^{2}\right)$

for $\mathbf{U}^{1} \in V, \quad \forall \mathbf{v}^{2} \in V$,

$a\left(\mathbf{U}^{2}, \mathbf{v}^{1}\right)-\omega^{2} b\left(\mathbf{U}^{2}, \mathbf{v}^{1}\right)=L^{2}\left(\mathbf{v}^{1}\right)$,

for $\mathbf{U}^{2} \in V, \quad \forall \mathbf{v}^{1} \in V$.
Next, we derive the sensitivity of mutual mean compliance $L^{2}\left(\mathbf{U}^{1}\right)$ with respect to a design variable $A$. This sensitivity is employed in the optimization procedure. Substituting $\mathbf{U}^{2}$ into $\mathbf{v}^{2}$ in (14) and $\mathbf{U}^{1}$ into $\mathbf{v}^{1}$ in (15), we obtain the following relation at equilibrium:

$L^{1}\left(\mathbf{U}^{2}\right)=a\left(\mathbf{U}^{1}, \mathbf{U}^{2}\right)-\omega^{2} b\left(\mathbf{U}^{1}, \mathbf{U}^{2}\right)=L^{2}\left(\mathbf{U}^{1}\right)$.

Taking the first variation of $L^{2}\left(\mathbf{U}^{1}\right)$ with respect to $\mathbf{U}^{1}$, $\mathbf{U}^{2}$ and $A$, using (16) yields

$\delta L^{2}\left(\mathbf{U}^{1}\right)=-\left[\delta a\left(\mathbf{U}^{1}, \mathbf{U}^{2}\right)-\omega^{2} \delta b\left(\mathbf{U}^{1}, \mathbf{U}^{2}\right)-\delta L^{1}\left(\mathbf{U}^{2}\right)-\right.$

$\left.\delta L^{2}\left(\mathbf{U}^{1}\right)\right]=-\left[a\left(\mathbf{U}^{2}, \frac{\partial \mathbf{U}^{1}}{\partial A} \delta A+\delta \mathbf{U}^{1}\right)-\right.$

$\left.\omega^{2} b\left(\mathbf{U}^{2}, \frac{\partial \mathbf{U}^{1}}{\partial A} \delta A+\delta \mathbf{U}^{1}\right)-L^{2}\left(\frac{\partial \mathbf{U}^{1}}{\partial A} \delta A+\delta \mathbf{U}^{1}\right)\right]-$

$\left[a\left(\mathbf{U}^{1}, \frac{\partial \mathbf{U}^{2}}{\partial A} \delta A+\delta \mathbf{U}^{2}\right)-\omega^{2} b\left(\mathbf{U}^{1}, \frac{\partial \mathbf{U}^{2}}{\partial A} \delta A+\delta \mathbf{U}^{2}\right)-\right.$

$\left.L^{1}\left(\frac{\partial \mathbf{U}^{2}}{\partial A} \delta A+\delta \mathbf{U}^{2}\right)\right]-\int_{\Omega} \varepsilon\left(\mathbf{U}^{2}\right)^{T} \frac{\partial \mathbf{E}}{\partial A} \varepsilon\left(\mathbf{U}^{1}\right) \mathrm{d} \Omega \delta A+$

$\omega^{2} \int_{\Omega} \frac{\partial \varrho}{\partial A} \mathbf{U}^{1} \bullet \mathbf{U}^{2} \mathrm{~d} \Omega \delta A$

Since the following relations are obtained using (14) and (15):

$a\left(\mathbf{U}^{1}, \frac{\partial \mathbf{U}^{2}}{\partial A} \delta A+\delta \mathbf{U}^{2}\right)-\omega^{2} b\left(\mathbf{U}^{1}, \frac{\partial \mathbf{U}^{2}}{\partial A} \delta A+\delta \mathbf{U}^{2}\right)-$

$L^{1}\left(\frac{\partial \mathbf{U}^{2}}{\partial A} \delta A+\delta \mathbf{U}^{2}\right)=0$,

$a\left(\mathbf{U}^{2}, \frac{\partial \mathbf{U}^{1}}{\partial A} \delta A+\delta \mathbf{U}^{1}\right)-\omega^{2} b\left(\mathbf{U}^{2}, \frac{\partial \mathbf{U}^{1}}{\partial A} \delta A+\delta \mathbf{U}^{1}\right)-$

$L^{2}\left(\frac{\partial \mathbf{U}^{1}}{\partial A} \delta A+\delta \mathbf{U}^{1}\right)=0$

we obtain the sensitivity of $L^{2}\left(\mathbf{U}^{1}\right)$ with respect to $A$ as follows:

$\frac{\partial L^{2}\left(\mathbf{U}^{1}\right)}{\partial A}=-\int_{\Omega} \varepsilon\left(\mathbf{U}^{2}\right)^{T} \frac{\partial \mathbf{E}}{\partial A} \varepsilon\left(\mathbf{U}^{1}\right) \mathrm{d} \Omega+$

$\omega^{2} \int_{\Omega} \frac{\partial \varrho}{\partial A} \mathbf{U}^{1} \bullet \mathbf{U}^{2} \mathrm{~d} \Omega$. 
Next, we only consider Case (a). The linear form implying the mean compliance in the dynamic problem is defined by

$$
L^{1}\left(\mathbf{U}^{1}\right)=\int_{\Gamma_{t^{1}}} \mathbf{T}^{1} \bullet \mathbf{U}^{1} \mathrm{~d} \Gamma=\int_{\Gamma_{t^{1}}} T_{i}^{1} U_{i}^{1} \mathrm{~d} \Gamma, \quad \mathbf{U}^{1} \in V .
$$

This mean compliance was first introduced by Ma et al. (1995). Mean compliance $L^{1}\left(\mathbf{U}^{1}\right)$ is interpreted as the measure of stiffness at boundary $\Gamma_{t^{1}}$, when we apply boundary traction $\mathbf{t}^{1}$ at boundary $\Gamma_{t^{1}}$. By minimizing or decreasing the absolute value of $L^{1}\left(\mathbf{U}^{1}\right)$, we can obtain sufficient stiffness along a direction specified by $\mathbf{T}^{1}$. Furthermore, following the derivation of the sensitivity of mutual mean compliance $L^{2}\left(\mathbf{U}^{1}\right)$ for this case, we obtain the sensitivity of mean compliance $L^{1}\left(\mathbf{U}^{1}\right)$ with respect to a design variable $A$ as follows:

$$
\begin{aligned}
& \frac{\partial L^{1}\left(\mathbf{U}^{1}\right)}{\partial A}=-\int_{\Omega} \varepsilon\left(\mathbf{U}^{1}\right)^{T} \frac{\partial \mathbf{E}}{\partial A} \varepsilon\left(\mathbf{U}^{1}\right) \mathrm{d} \Omega+ \\
& \omega^{2} \int_{\Omega} \frac{\partial \varrho}{\partial A} \mathbf{U}^{1} \bullet \mathbf{U}^{1} \mathrm{~d} \Omega .
\end{aligned}
$$

\section{4}

\section{Formulation of the multi-optimization problem}

In this section, we formulate the multi-optimization problem in order to design a flexible structure under a periodic load in time. Suppose that the original design domain, $\Omega_{d}$, of a flexible structure is fixed at boundary $\Gamma_{d}$ and is subjected to the periodically oscillating boundary traction $\mathbf{t}^{1}=\mathbf{T}^{1} e^{j \omega t}$, where $\omega$ is an excitation frequency and $t$ is time, as shown in Fig. 3. We also consider an extended design domain $D$ including $\Omega_{d}$.

Now, we design a flexible structure which starts to deform along a direction specified by unit dummy vector $\mathbf{T}^{2}$, where $\mathbf{t}^{2}=\mathbf{T}^{2} e^{j \omega t}$ as shown in Fig. 3a. To implement this function in the flexible structure, we must take into account two requirements: the kinematic requirement and the structural requirement, as Frecker et al. (1997) explained. The kinematic requirement is identical to our design specification mentioned above. To satisfy the kinematic requirement, the flexible structure must have sufficient flexibility, which provides sufficient deformation along a direction specified by unit dummy vector $\mathbf{T}^{2}$ when periodically oscillating boundary traction $\mathbf{t}^{1}$ is applied. The kinematic requirement is obtained by maximizing the absolute value of mutual mean compliance $L^{2}\left(\mathbf{U}^{1}\right)$ defined by (8). On the other hand, for the structural requirement, we impose sufficient stiffness at boundaries $\Gamma_{t^{1}}$ and $\Gamma_{t^{2}}$ as shown in Figs. 3b and c. This sufficient stiffness works to maintain the shape of the flexible structure when traction $\mathbf{t}^{1}$ is applied in Case (b), and the reaction force imposed by an object such as a workpiece is applied as shown in Case (c). For Case (b), sufficient stiffness is obtained by minimizing the absolute value of the mean compliance at boundary $\Gamma_{t^{1}}$, posed by traction $\mathbf{t}^{1}$ while boundary $\Gamma_{t^{2}}$ is fixed, since the flexible structure is supposed to be imposed by the reaction force of the object. For Case (c), sufficient stiffness is obtained by minimizing the mean compliance at boundary $\Gamma_{t^{2}}$ posed by the reaction force while boundary $\Gamma_{t}$ is fixed because the flexible structure must be imposed by applied traction $\mathbf{t}^{1}$. The direction of the reaction force is assumed to be opposite to that of dummy load vector $\mathbf{T}^{2}$. However, the excitation frequency of the reaction force is not uniquely determined, a priori, and is usually unknown in the design phase of some flexible structures, since this depends on the contact condition between the flexible structure and the object. In this case, we assume that the excitation frequency of the reaction force is zero in the engineering sense. That is, the reaction force is considered to be statically applied. This is because the implementation of the sufficient stiffness in the static case can provide approximately the same stiffness in the dynamic case in the average sense if the excitation frequency is not specified due to the contact condition. However, we specify the excitation frequency if this is known in the design phase. A typical flexible structure design in which we know the excitation frequency is the resonator design. In this case, the excitation frequency of the reaction force is the same as that of the applied traction.

Thus, the optimization problem for flexible structure design under a periodic load is formulated as follows:

$$
\begin{aligned}
& \max _{\alpha, \beta, \theta}\left|L^{2}\left(\mathbf{U}^{1}\right)\right|=\left|\int_{\Gamma_{t^{2}}} \mathbf{T}^{2} \bullet \mathbf{U}^{1} \mathrm{~d} \Gamma\right|, \\
& \min _{\alpha, \beta, \theta}\left|L^{3}\left(\mathbf{U}^{3}\right)\right|=\left|\int_{\Gamma_{t^{1}}} \mathbf{T}^{3} \bullet \mathbf{U}^{3} \mathrm{~d} \Gamma\right|, \\
& \min _{\alpha, \beta, \theta}\left|L^{4}\left(\mathbf{U}^{4}\right)\right|=\left|\int_{\Gamma_{t^{2}}} \mathbf{T}^{4} \bullet \mathbf{U}^{4} \mathrm{~d} \Gamma\right|,
\end{aligned}
$$

subject to

$0 \leq \alpha \leq 1$

$0 \leq \beta \leq 1$,

$\mathbf{T}^{3}=\mathbf{T}^{1}$

$\mathbf{T}^{4}=-\mathbf{T}^{2}$,

$a\left(\mathbf{U}^{1}, \mathbf{v}^{1}\right)-\omega^{2} b\left(\mathbf{U}^{1}, \mathbf{v}^{1}\right)=L^{1}\left(\mathbf{v}^{1}\right)$,

for $\mathbf{U}^{1} \in V^{(a)}, \quad \forall \mathbf{v}^{1} \in V^{(a)}$, 


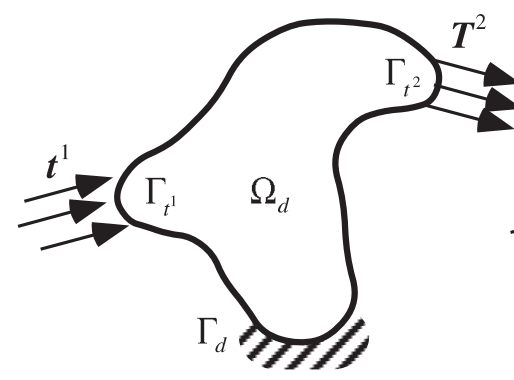

Case (a)

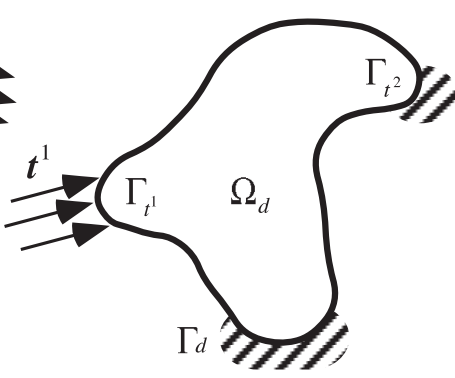

Case (b)

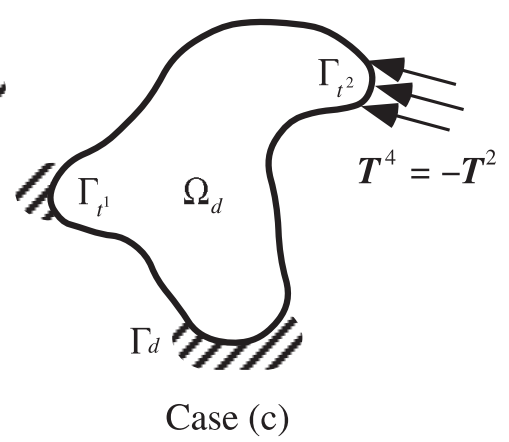

Fig. 3 Specifications for a flexible structure design

$$
\begin{aligned}
& a\left(\mathbf{U}^{2}, \mathbf{v}^{2}\right)-\omega^{2} b\left(\mathbf{U}^{2}, \mathbf{v}^{2}\right)=L^{2}\left(\mathbf{v}^{2}\right), \\
& \text { for } \mathbf{U}^{2} \in V^{(a)}, \quad \forall \mathbf{v}^{2} \in V^{(a)}, \\
& a\left(\mathbf{U}^{3}, \mathbf{v}^{3}\right)-\omega^{2} b\left(\mathbf{U}^{3}, \mathbf{v}^{3}\right)=L^{3}\left(\mathbf{v}^{3}\right), \\
& \text { for } \mathbf{U}^{3} \in V^{(b)}, \quad \forall \mathbf{v}^{3} \in V^{(b)}, \\
& a\left(\mathbf{U}^{4}, \mathbf{v}^{4}\right)-\omega_{R}^{2} b\left(\mathbf{U}^{4}, \mathbf{v}^{4}\right)=L^{4}\left(\mathbf{v}^{4}\right), \\
& \text { for } \mathbf{U}^{4} \in V^{(c)}, \quad \forall \mathbf{v}^{4} \in V^{(c)}, \\
& g(\alpha, \beta)=\int_{\Omega_{d}}(1-\alpha \beta) \mathrm{d} \Omega-\Omega_{s} \leq 0,
\end{aligned}
$$

where $V^{(a)}=\left\{\mathbf{v}=v_{i} \mathbf{e}_{i}: v_{i} \in H^{1}(D)\right.$ with $\mathbf{v}=0$ on $\left.\Gamma_{d}\right\}$, $V^{(b)}=\left\{\mathbf{v}=v_{i} \mathbf{e}_{i}: v_{i} \in H^{1}(D)\right.$ with $\mathbf{v}=0$ on $\Gamma_{d}$ and $\left.\Gamma_{t^{2}}\right\}$, and $V^{(c)}=\left\{\mathbf{v}=v_{i} \mathbf{e}_{i}: v_{i} \in H^{1}(D)\right.$ with $\mathbf{v}=0$ on $\Gamma_{d}$ and $\left.\Gamma_{t^{1}}\right\} . \Omega_{s}$ is the total volume constraint of the solid material forming the porous structure, and $\omega_{R}$ is the excitation frequency of the reaction force imposed by the object.

Several methods have been developed to deal with multi-objective problems such as the optimization problem defined by (23) through (32) (see e.g. Stadler 1988; Koski 1993). Among them, the weighting method has been employed most commonly because of its convenience of formulation. In this method, the multi-objective function is formulated as the summation of objective functions with the weighting coefficients. Using this multi-objective function, we can obtain all the Pareto optima by changing the weighting coefficients in the case where the Pareto optima are convex with respect to design variables, as Koski (1985) explained. However, from the physical point of view, all optimal solutions on the Pareto curve are not appropriate in the specific optimization problem formulated by (23) through (32). This is because mutual mean compliance $L^{2}\left(\mathbf{U}^{1}\right)$ can be close to infinite with certain weighting coefficients. In this case, the optimal configuration can have full voids, discon- nected structure, or ambiguous structure in the design domain with higher flexibility. Therefore, we must employ a different multi-objective function in order to seek the appropriate optimal configuration in the Pareto optima. In this study, the following objective function is proposed:

$$
\begin{aligned}
& \max _{\alpha, \beta, \theta} f_{1}=W \log \left|L^{2}\left(\mathbf{U}^{1}\right)\right|- \\
& \frac{1}{2}(1-W) \log \left[w_{s} L^{3}\left(\mathbf{U}^{3}\right)^{2}+\left(1-w^{s}\right) L^{4}\left(\mathbf{U}^{4}\right)^{2}\right],
\end{aligned}
$$

where $W$ and $w_{s}$ are the weighting coefficients such that $0 \leq W \leq 1$ and $0 \leq w_{s} \leq 1$. $W$ represents the relative importance of flexibility and stiffness. $w_{s}$ represents the relative importance of two stiffnesses defined in Case (b) and Case (c) shown in Fig. 3. We take squares of two mean compliances $L^{3}\left(\mathbf{U}^{3}\right)$ and $L^{4}\left(\mathbf{U}^{4}\right)$ in (33) since the absolute values of them must be minimized to obtain sufficient stiffness in the dynamics case.

Taking a first variation of $f_{1}$ yields

$$
\begin{aligned}
& \delta f_{1}=W \frac{\delta L^{2}\left(\mathbf{U}^{1}\right)}{L^{2}\left(\mathbf{U}^{1}\right)}-(1-W) \times \\
& \frac{w_{s} L^{3}\left(\mathbf{U}^{3}\right) \delta L^{3}\left(\mathbf{U}^{3}\right)+\left(1-w_{s}\right) L^{4}\left(\mathbf{U}^{4}\right) \delta L^{4}\left(\mathbf{U}^{4}\right)}{w_{s} L^{3}\left(\mathbf{U}^{3}\right)^{2}+\left(1-w_{s}\right) L^{4}\left(\mathbf{U}^{4}\right)^{2}} .
\end{aligned}
$$

This implies that since the equivalent weighting coefficient of the small perturbation, $\delta L^{2}\left(\mathbf{U}^{1}\right)$, is $W / L^{2}\left(\mathbf{U}^{1}\right)$, it is proportional to the inverse of $L^{2}\left(\mathbf{U}^{1}\right)$. Therefore, as $L^{2}\left(\mathbf{U}^{1}\right)$ increases toward infinity, the multi-objective function defined by (33) provides $L^{2}\left(\mathbf{U}^{1}\right)$ with the smaller value as the weighting coefficient, and as an effect we can obtain an appropriate configuration by setting $W$ to an appropriate value. Note that since weighting coefficient $W$ depends on excitation frequency $\omega$, we must adjust this weighting coefficient for a different frequency problem. This is because the mutual mean compliance has the relation with inertia part $\omega b\left(\mathbf{U}^{1}, \mathbf{U}^{2}\right)$ as shown by (16), and the effect caused by this inertia part depends on the 
excitation frequency $\omega$. It is also noted that since the multi-objective function defined by (33) is composed of the logarithm functions, the optimal solution obtained by (33) is one of the Pareto optima (see details by Nishiwaki et al. 1998).

\section{5}

\section{Numerical implementation}

Figure 4 shows a flowchart of the optimization procedure. There are five steps in the per-iteration loop of the optimization.

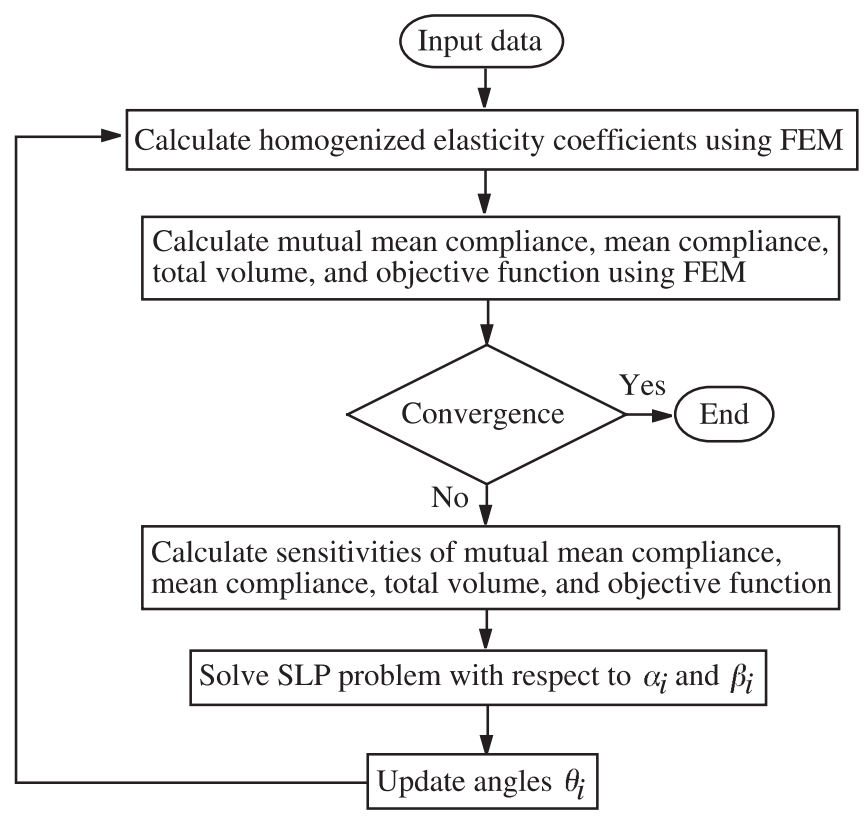

Fig. 4 Flowchart of optimization procedure

In the first step, homogenized elasticity tensor $\mathbf{E}^{H}$ is computed using the finite element method (see details by Guedes and Kikuchi 1988). Using the finite element method, the numerical values of homogenized elasticity tensor $\mathbf{E}^{H}$ are calculated at six discretized points between 0 and 1 of microscopic design variables $\alpha$ and $\beta$. Next, $\mathbf{E}^{H}$ is interpolated using Bezier curves. Note that homogenized mass density $\varrho^{H}$ is analytically obtained by (6).

In the second step, the mutual mean compliance, the two mean compliances, the total volume, and the objective function are computed using FEM. Extended design domain $D$ is discretized by the finite elements. We approximate that the configuration of the microstructure is uniform in each element. That is, the configuration of the microstructure in the $i$-th element can be represented by three design variables, $\alpha_{i}, \beta_{i}$, and $\theta_{i}$ for $i=1, \ldots, n$, where $n$ is the number of elements. Therefore, the total number of design variables is $3 n$ in the entire design domain $D$. Microscopic design variables $\alpha_{i}$ and $\beta_{i}$ in each element are bounded as follows in order to avoid singularity in the $\mathrm{FE}$ analysis although these design variables are theoretically bounded by (24) and (25):

$0 \leq \alpha_{i} \leq \alpha_{\text {upp }}<1$

$0 \leq \beta_{i} \leq \beta_{\text {upp }}<1$

where $\alpha_{\text {upp }}$ and $\beta_{\text {upp }}$ are the upper bounds of variables $\alpha_{i}$ and $\beta_{i}$, respectively. These are specified as sufficiently large numbers, but less than one. In the FE analysis, four node isoparametric full integration elements with bilinear shape functions are used.

In the third step, sensitivities of mutual compliance, the two mean compliances, and total volume, and the objective function with respect to design variables are computed if the objective function is not converged. Note that sensitivities of mutual compliance and two mean compliances are obtained by (20) and (22).

In the fourth step, the optimization problem with $\alpha_{i}$ and $\beta_{i}$ is solved by sequential linear programming (SLP). The optimality criteria method is used in topology optimization (e.g. Suzuki and Kikuchi 1991; Ma et al. 1995) because this method can deal with a large number of design variables and has relatively fast convergence. However, in this method, the heuristics determining the update rule must be adequately adjusted for different objective optimization problems. On the other hand, SLP can deal with a variety of objective functions and can handle numerous design variables although fast convergence cannot be expected. Thus, SLP is employed as an optimizer in the research. In SLP, the linearized problem in the small area specified by move limits is solved using linear programming. Move limit $\Delta A$ for a design variable $A$ is set as follows to avoid the locking of a design variable close to zero (see details by Nishiwaki et al. 1998):

$\Delta A=\max \left(\boldsymbol{\xi} A, \Delta A_{\min }\right)$,

where $\boldsymbol{\xi}$ is a constant ratio set to 0.1 , and $\Delta A_{\min }$ is a minimum move limit set to $1.0 \%$ of the maximum value of the design variable. In the linearized optimization problem, a package of the simplex method, DSPLP from the SLATEC library (Hanson and Hibert 1981), is used.

In the fifth step, angle $\theta_{i}$ is updated. In this study, this angle is practically updated to minimize the two mean compliances for simplicity of computation. To minimize the two mean compliances, the direction of angle $\theta_{i}$ is updated to the principal direction of stress (see details by Pedersen 1989) using the multi-loading criterion which was proposed by Suzuki and Kikuchi (1991). That is, the angle is updated by the following equation:

$\min _{\theta} \max \left[L^{3}\left(\mathbf{U}^{3}\right), L^{4}\left(\mathbf{U}^{4}\right)\right]$. 
6

\section{Numerical examples}

In this section, two examples in the two-dimensional case are presented to examine the optimal configurations. The properties of the isotropic material correspond to Young's modulus $=100$, Poisson's ratio $=0.3$, and mass density $=$ $7.85 \times 10^{-6}$. The magnitude of amplitude of an applied force is assumed to be a unit load.

\section{1}

\section{Example 1}

Figure 5 shows the design domain where boundary conditions and specifications are as indicated. The left-hand side boundary is fixed to support the flexible structure. The deformation along a direction specified by dummy load $\mathbf{F}^{2}$ at point $P_{2}$ is to be maximized when the periodically oscillating force along a direction specified by $\mathbf{F}^{1}$ is applied at point $P_{1}$, while the stiffnesses at both points $P_{1}$ and $P_{2}$ are to be maximized. That is, the absolute value of the mutual mean compliance, $L^{2}\left(\mathbf{U}^{1}\right)$, defined by two loads, is to be maximized for the kinematic requirement, while the absolute values of the mean compliance, $L^{3}\left(\mathbf{U}^{3}\right)$, at point $P_{1}$ and the mean compliance, $L^{4}\left(\mathbf{U}^{4}\right)$, at point $P_{2}$ are to be minimized for the structural requirement. Loads $\mathbf{F}^{1}$ and $\mathbf{F}^{2}$ correspond to tractions $\mathbf{T}^{1}$ and $\mathbf{T}^{2}$, respectively.

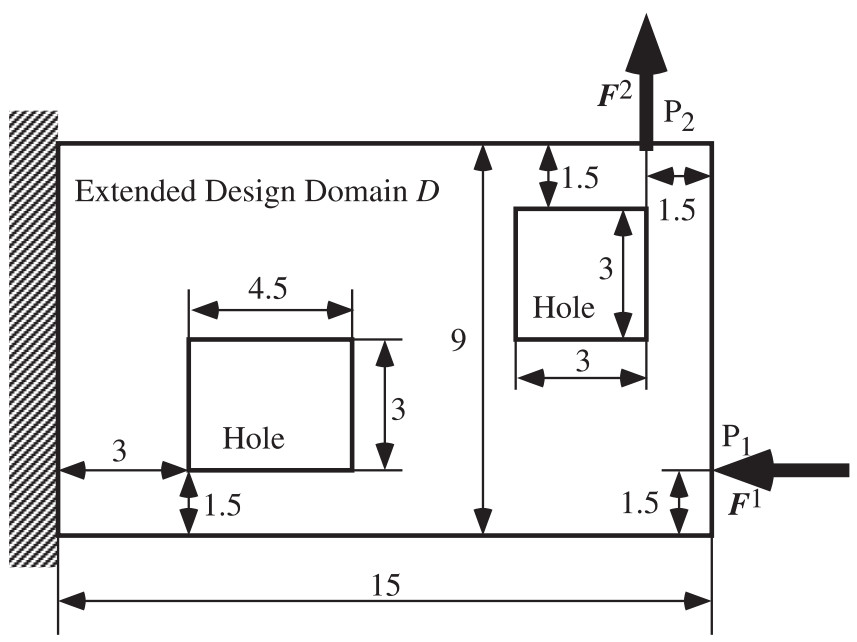

Fig. 5 Design domain for a simple flexible structure

The design domain is discretized using 1250 finite elements. The total volume constraint of the material $\Omega_{s}$ is considered to be 22.5 , which is $20 \%$ of the volume of the whole design domain. The weighting coefficient, $w_{s}$, in (33) is set to 0.4 . The excitation frequency of the reaction force, $\omega_{R}$, is assumed to be zero. The optimal configurations for 10 different excitation frequency cases are computed: excitation frequency $\omega=0(\mathrm{rad} / \mathrm{s})(0 \mathrm{~Hz}), 502.65$ $(\mathrm{rad} / \mathrm{s})(80 \mathrm{~Hz}), 753.98(\mathrm{rad} / \mathrm{s})(120 \mathrm{~Hz}), 1005.31(\mathrm{rad} / \mathrm{s})$ $(160 \mathrm{~Hz}), 1256.64(\mathrm{rad} / \mathrm{s})(200 \mathrm{~Hz}), 1507.96(\mathrm{rad} / \mathrm{s})(240$ $\mathrm{Hz}), 1759.29(\mathrm{rad} / \mathrm{s})(280 \mathrm{~Hz}), 2010.62(\mathrm{rad} / \mathrm{s})(320 \mathrm{~Hz})$, $2261.95(\mathrm{rad} / \mathrm{s})(360 \mathrm{~Hz})$, and $2513.27(\mathrm{rad} / \mathrm{s})(400 \mathrm{~Hz})$.

There exist many local optima in this dynamic problem since we can achieve the same eigenfrequency with different material distributions in the fixed design domain in order to obtain sufficient flexibility. We must choose appropriate initial configurations in order to obtain the appropriate optimal configurations which have physical meaning among local optima. Therefore, we basically use the optimal configuration in the static case as an initial configuration in the dynamic case. That is, first the optimal configuration in the case of excitation frequency $\omega=0(\mathrm{rad} / \mathrm{s})(0 \mathrm{~Hz})$ is obtained using the uniform initial configuration where values of microscopic design variables $\alpha_{i}$ and $\beta_{i}$ are set to 0.90 , and the value of $\theta_{i}$ is set to 0.0 for $i=1, \ldots$, the number of elements (in all elements). Next, the optimal configurations in the case of $\omega=502.65(\mathrm{rad} / \mathrm{s})(80 \mathrm{~Hz})$ and $753.98(\mathrm{rad} / \mathrm{s})(120 \mathrm{~Hz})$ are obtained using the optimal configuration in the case of $\omega=0(\mathrm{rad} / \mathrm{s})(0 \mathrm{~Hz})$ as an initial configuration. The optimal configurations in the case of $\omega=1005.31(\mathrm{rad} / \mathrm{s})(160 \mathrm{~Hz}) \sim 1507.96(\mathrm{rad} / \mathrm{s})$ $(240 \mathrm{~Hz})$ are obtained using the optimal configuration in the case of $\omega=753.98(\mathrm{rad} / \mathrm{s})(120 \mathrm{~Hz})$ as an initial configuration. The optimal configurations in the case of $\omega=1759.29(\mathrm{rad} / \mathrm{s})(280 \mathrm{~Hz})$ and $2010.62(\mathrm{rad} / \mathrm{s})$ $(320 \mathrm{~Hz})$ are obtained using the optimal configuration in the case of $\omega=1507.96(\mathrm{rad} / \mathrm{s})(240 \mathrm{~Hz})$ as an initial configuration. The optimal configurations in the case of $\omega=2261.95(\mathrm{rad} / \mathrm{s})(360 \mathrm{~Hz})$ and $2513.27(\mathrm{rad} / \mathrm{s})$ $(400 \mathrm{~Hz})$ are obtained using the optimal configuration in the case of $\omega=2010.62(\mathrm{rad} / \mathrm{s})(320 \mathrm{~Hz})$ as an initial configuration. Table 1 shows the weighting coefficient, $W$ in (33), set in the optimization and the lowest eigenfrequencies of the optimal configurations.

From Table 1, we can see that each optimal configuration has the lowest eigenfrequency which is almost the same as the excitation frequency of the applied force. It

Table 1 Weighting coefficient $W$ in (33) and the lowest eigenfrequencies of the optimal configurations $\left[\Omega_{s}=22.5\right.$ $\left.(20 \%), w_{s}=0.4\right]$

\begin{tabular}{llll}
\hline$\omega / 2 \pi(\mathrm{Hz})$ & $\begin{array}{l}\text { Initial } \\
\text { configuration }\end{array}$ & $W$ & $\begin{array}{l}\text { Lowest eigen- } \\
\text { frequency }(\mathrm{Hz})\end{array}$ \\
\hline \hline 0 & uniform & 0.5 & 29.39 \\
80 & $0 \mathrm{~Hz}$ & 0.05 & 80.40 \\
120 & $0 \mathrm{~Hz}$ & 0.05 & 119.28 \\
160 & $120 \mathrm{~Hz}$ & 0.1 & 159.91 \\
200 & $120 \mathrm{~Hz}$ & 0.2 & 200.32 \\
240 & $120 \mathrm{~Hz}$ & 0.3 & 239.78 \\
280 & $240 \mathrm{~Hz}$ & 0.4 & 280.80 \\
320 & $240 \mathrm{~Hz}$ & 0.3 & 321.26 \\
360 & $320 \mathrm{~Hz}$ & 0.3 & 359.62 \\
400 & $320 \mathrm{~Hz}$ & 0.3 & 400.07 \\
\hline
\end{tabular}




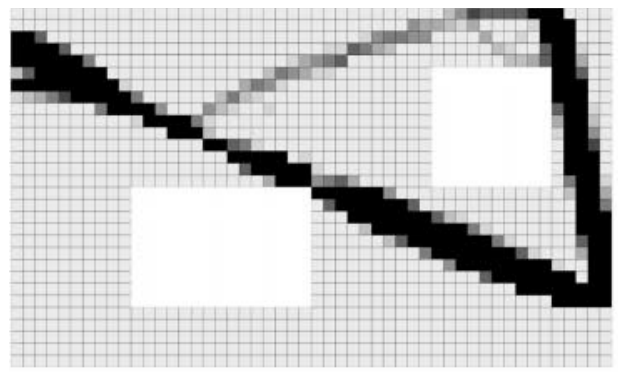

(a) $\omega=0.0(\mathrm{rad} / \mathrm{s})(0 \mathrm{~Hz}), W=0.5$

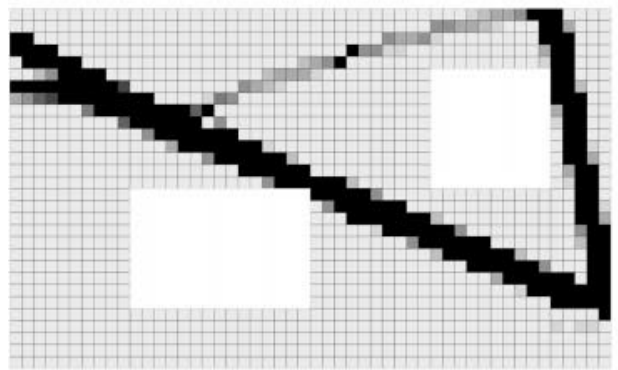

(c) $\omega=753.98(\mathrm{rad} / \mathrm{s})(120 \mathrm{~Hz}), W=0.05$

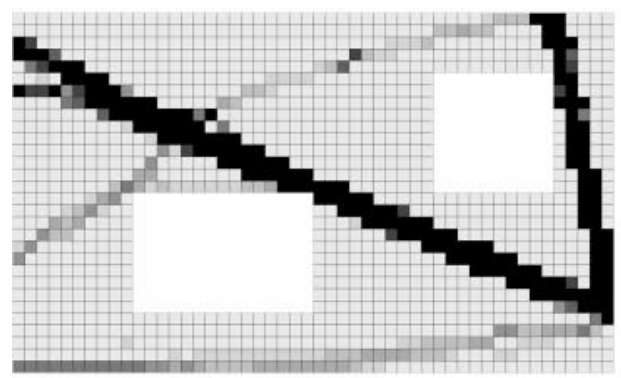

(e) $\omega=1256.64(\mathrm{rad} / \mathrm{s})(200 \mathrm{~Hz}), W=0.2$

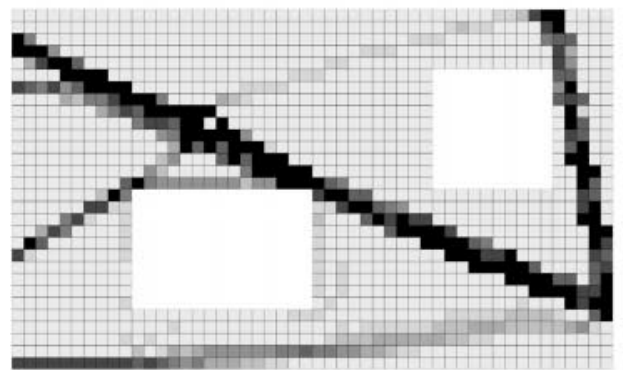

(g) $\omega=1759.29(\mathrm{rad} / \mathrm{s})(280 \mathrm{~Hz}), W=0.4$

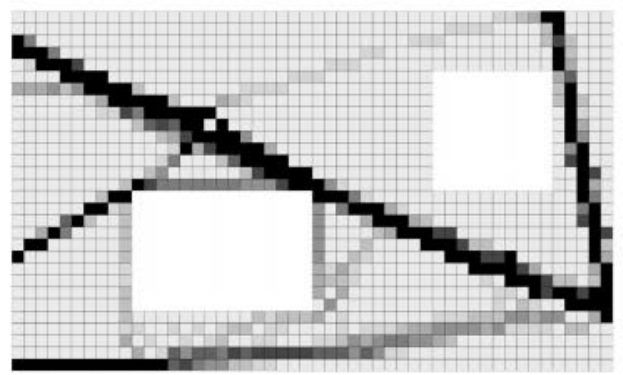

(i) $\omega=2261.95(\mathrm{rad} / \mathrm{s})(360 \mathrm{~Hz}), W=0.3$

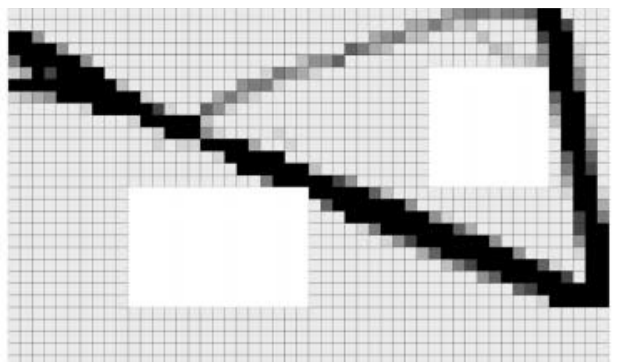

(b) $\omega=502.65(\mathrm{rad} / \mathrm{s})(80 \mathrm{~Hz}), W=0.05$

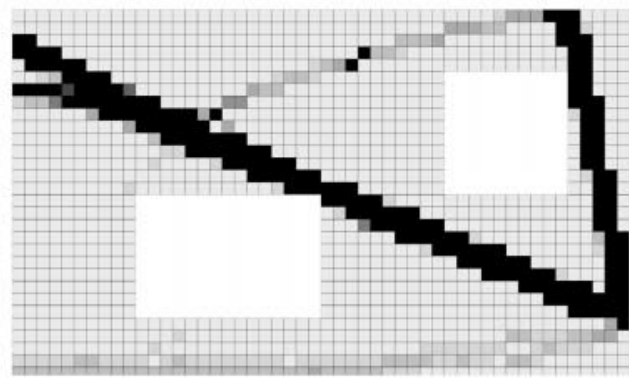

(d) $\omega=1005.31(\mathrm{rad} / \mathrm{s})(160 \mathrm{~Hz}), W=0.1$

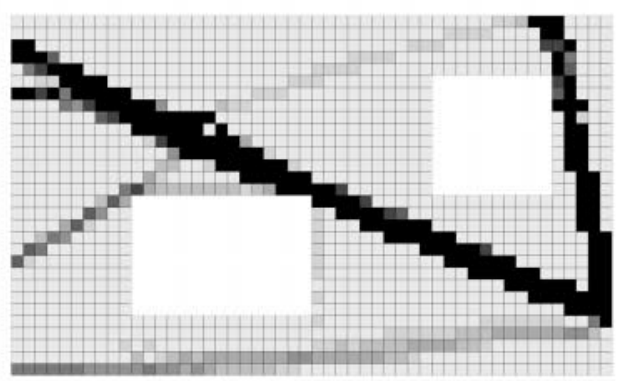

(f) $\omega=1507.96(\mathrm{rad} / \mathrm{s})(240 \mathrm{~Hz}), W=0.3$

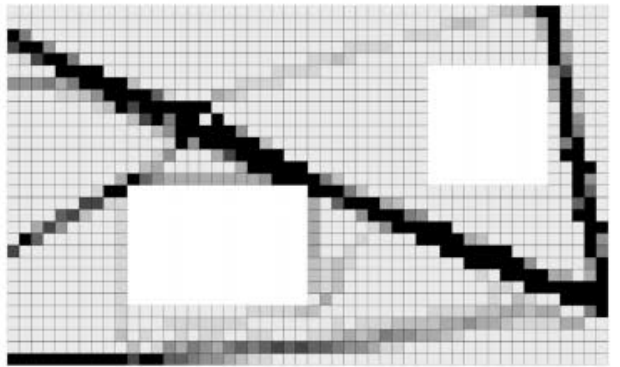

(h) $\omega=2010.62(\mathrm{rad} / \mathrm{s})(320 \mathrm{~Hz}), W=0.3$

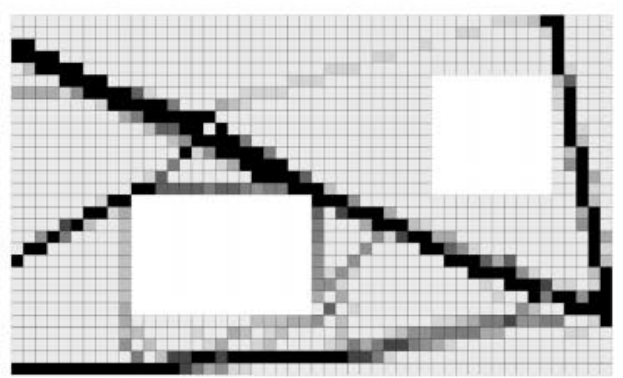

(j) $\omega=2513.27(\mathrm{rad} / \mathrm{s})(400 \mathrm{~Hz}), W=0.3$

Fig. 6 Optimal configurations of the simple flexible structure $\left[\Omega_{s}=22.5(20 \%), w_{s}=0.4\right]$ 
is reasonable that the resonance situation can provide the highest flexibility if we do not take into account the structural requirement. However, due to the structural requirement which prevents the objective function defined by (33) from going toward infinity, the lowest eigenfrequency is not exactly the same as the excitation frequency of the applied force. Figure 6 shows the optimal configurations for 10 different excitation frequency cases. It is clear that the optimal configuration depends on the excitation frequency. That is, the dynamic effect affects the topology configuration, especially in the high excitation frequency case. Therefore, the dynamic effect must be considered in the design phase if the excitation frequency of the applied force is high.

\section{2}

\section{Example 2}

We can design the resonator using the method presented here since the flexible structure obtained by maximizing the mutual mean compliance with the specified excitation frequency of the applied force is equivalent to the flexible structure with the same lowest eigenfrequency as the specified excitation frequency. Next, we shall show the design of a simple resonator by maximizing the mutual mean compliance with the specified excitation frequency.

Figure 7 shows a half view of the design domain where boundary conditions and specifications are as indicated. As shown in this figure, the right-hand side boundary of the design domain is fixed to support the resonator, and the symmetry boundary condition is posed at the lefthand side boundary.

Here, we consider the resonance condition which has to occur along a direction specified by a dummy load $\mathbf{F}^{2}$ at point $P_{2}$, when the periodically oscillating force along a direction specified by $\mathbf{F}^{1}$ is applied at point $P_{1}$, while the resonator has sufficient stiffness at both points $P_{1}$ and $P_{2}$. In order to achieve this performance, the absolute value of the mutual mean compliance, $L^{2}\left(\mathbf{U}^{1}\right)$ defined by two loads, is to be maximized for the kinematic requirement, while the absolute values of the mean compliance, $L^{3}\left(\mathbf{U}^{3}\right)$ at point $P_{1}$, and the mean compliance, $L^{4}\left(\mathbf{U}^{4}\right)$ at point $P_{2}$, are to be minimized for the structural requirement. Loads $\mathbf{F}^{1}$ and $\mathbf{F}^{2}$ correspond to tractions $\mathbf{T}^{1}$ and $\mathbf{T}^{2}$, respectively.

The design domain is discretized using 800 finite elements. The total volume constraint of the material $\Omega_{s}$ is considered to be 240 , which is $30 \%$ of the volume of the whole design domain. The weighting coefficient, $w_{s}$ in (33), is set to 0.4 . The excitation frequency of the reaction force, $\omega_{R}$, is assumed to be the same as that of the applied force, $\omega$.

We must also choose appropriate initial configurations in order to have appropriate optimal configurations in this example. This is because there can exist many local optima in this problem and some of them do not have

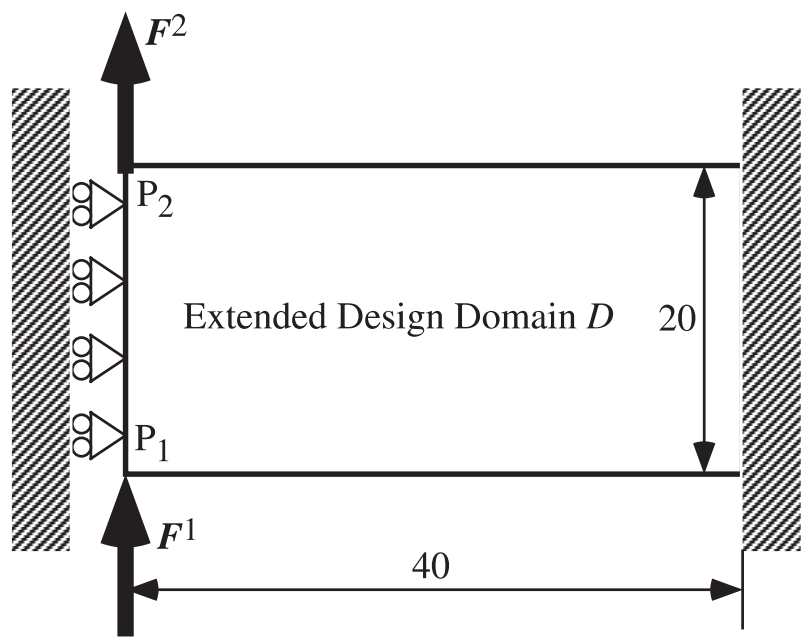

Fig. 7 Design domain for a simple resonators

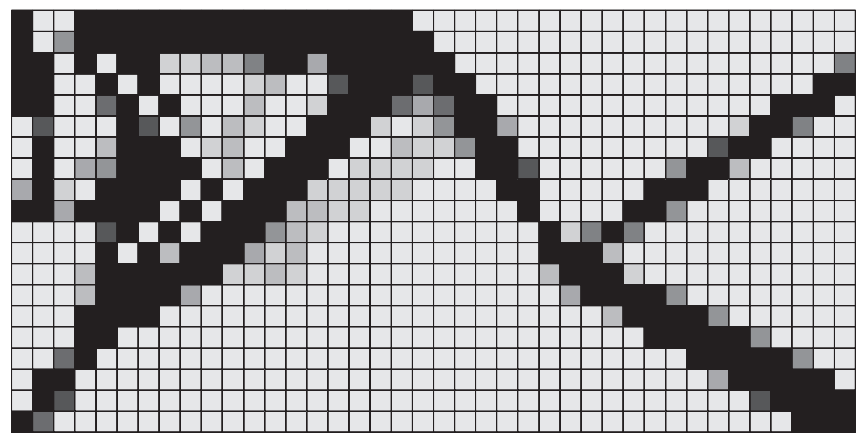

Fig. 8 Optimal configuration in the static case obtained by (39)

physical meaning or are rather trivial solutions which only have a rigid-body mode to achieve the performance described above. In this example, we first obtain the optimal configuration by minimizing the mean compliance where $\mathbf{F}^{1}$ is applied at point $P_{1}$, and point $P_{2}$ is not fixed, in addition to implementing the same specifications in the static case as that in the dynamic case in order to avoid a trivial solution. This configuration is used as the initial configuration for the resonator design. To achieve this performance, we use the following objective function instead of (33) where the excitation frequency $\omega$ is set to zero:

$\max _{\alpha, \beta, \theta} f_{2}=W \log \left|L^{2}\left(\mathbf{U}^{1}\right)\right|-\frac{1}{2}(1-W) \times$

$\log \left[w_{s} L^{3}\left(\mathbf{U}^{3}\right)^{2}+\left(1-w_{s}\right) L^{4}\left(\mathbf{U}^{4}\right)^{2}+w_{a} L^{5}\left(\mathbf{U}^{5}\right)^{2}\right]$,

where

$\mathbf{t}^{5}=\mathbf{T}^{1}$

$a\left(\mathbf{u}^{5}, \mathbf{v}^{5}\right)=L^{5}\left(\mathbf{u}^{5}\right), \quad$ for $\mathbf{u}^{5} \in V^{(a)}, \forall \mathbf{v}^{5} \in V^{(a)}$, 


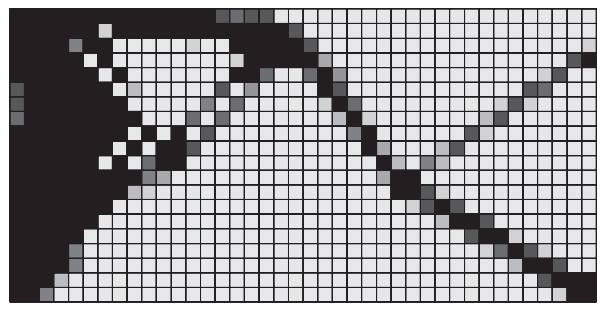

(a) $\omega=753.98(\mathrm{rad} / \mathrm{s})(120 \mathrm{~Hz}), W=0.01$

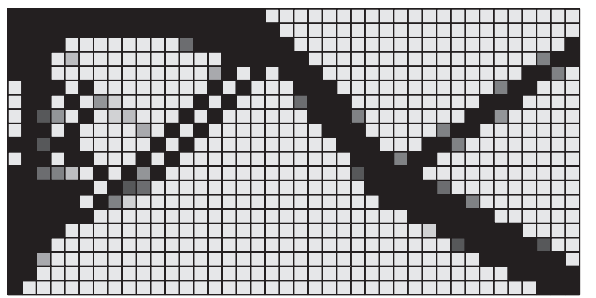

(c) $\omega=1256.64(\mathrm{rad} / \mathrm{s})(200 \mathrm{~Hz}), W=0.13$

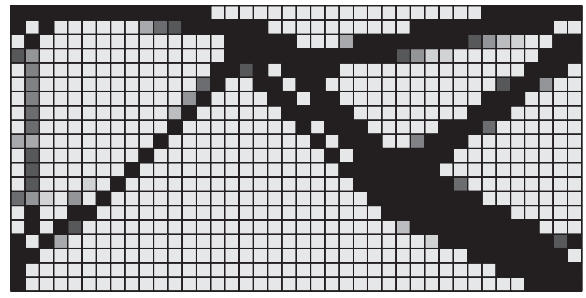

(e) $\omega=1759.29(\mathrm{rad} / \mathrm{s})(280 \mathrm{~Hz}), W=0.5$

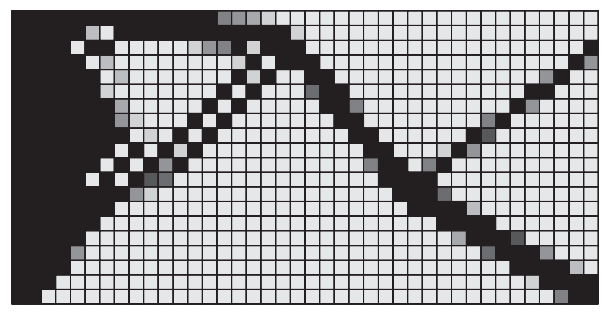

(b) $\omega=1005.31(\mathrm{rad} / \mathrm{s})(160 \mathrm{~Hz}), W=0.001$

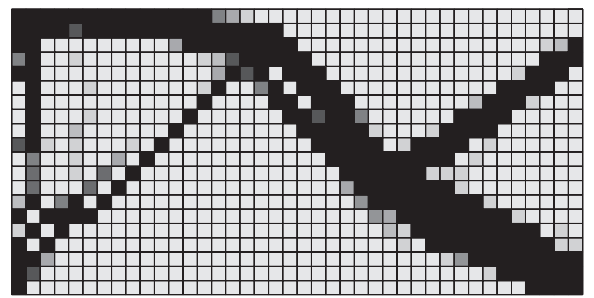

(d) $\omega=1507.96(\mathrm{rad} / \mathrm{s})(240 \mathrm{~Hz}), W=0.42$

Fig. 9 Optimal configurations of the resonator $\left[\Omega_{s}=240(30 \%), w_{s}=0.4\right)$

and weighting coefficient $w_{a}$ is set to 0.09 in this example. Using the uniform initial configuration where values of microscopic design variables $\alpha_{i}$ and $\beta_{i}$ are set to 0.90 and the value of $\theta_{i}$ is set to 0.0 for $i=1, \ldots$, the number of elements (in all elements), we obtain the optimal configuration in the static case as shown in Fig. 8. The optimal configurations for 5 different excitation frequency cases are computed by (33) using the optimal configuration in the static case as the initial configuration: excitation frequency $\omega=753.98(\mathrm{rad} / \mathrm{s})(120 \mathrm{~Hz}), 1005.31(\mathrm{rad} / \mathrm{s})$ $(160 \mathrm{~Hz}), 1256.64(\mathrm{rad} / \mathrm{s})(200 \mathrm{~Hz}), 1507.96(\mathrm{rad} / \mathrm{s})$ $(240 \mathrm{~Hz})$, and $1759.29(\mathrm{rad} / \mathrm{s})(280 \mathrm{~Hz})$. Table 2 shows the weighting coefficient, $W$ in (33), and the lowest eigenfrequencies of the optimal configurations in the resonator design.

From Table 2, we can see that each optimal configuration also has the lowest eigenfrequency which is almost the same as the excitation frequency of the applied force in this example. This means that we can change the resonance frequency by specifying a different excitation frequency. Note that the lowest eigenfrequency is not exactly the same as the excitation frequency of the applied force due to the structural requirement. However, this
Table 2 Weighting coefficient $W$ in (33) and the lowest eigenfrequencies of the optimal configurations $\left[\Omega_{s}=22.5\right.$ $\left.(20 \%), w_{s}=0.4\right]$

\begin{tabular}{lll}
\hline$\omega / 2 \pi(\mathrm{Hz})$ & $W$ & Lowest eigenfrequency $(\mathrm{Hz})$ \\
\hline \hline 0 & 0.49 & 52.00 \\
120 & 0.01 & 121.32 \\
160 & 0.001 & 160.11 \\
200 & 0.13 & 200.17 \\
240 & 0.43 & 239.90 \\
280 & 0.5 & 280.41 \\
\hline
\end{tabular}

difference is negligible in the engineering sense. Figure 9 shows the optimal configurations for 5 different excitation frequency cases. It is clear that the optimal configuration changes as the excitation frequency is changed. This means that the dynamic effect must be taken into account appropriately in the resonator design.

Figure 10 shows the eigenmode of the lowest eigenfrequency of the optimal configuration in the case of $200 \mathrm{~Hz}$. We can see that the eigenmode matches the specified res- 


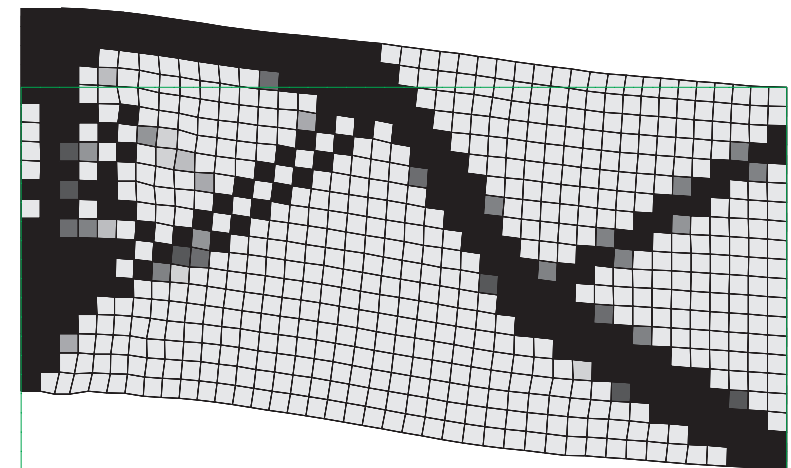

Fig. 10 The eigenmode of the lowest eigenfrequency $(200 \mathrm{~Hz})$

onance condition. Therefore, the resonance occurs along a direction specified by a dummy load $\mathbf{F}^{2}$ at point $P_{2}$. Thus, we confirm that we can also design the resonator using the method presented here.

\section{7}

\section{Conclusions}

In this research, we developed a topology optimization method implementing flexibility for cases in which the boundary is under a periodic load in time. First, the mutual mean compliance was formulated using the mutual energy concept in order to define the measure of flexibility in the dynamic case. The sensitivity of the mutual mean compliance with respect to a design variable was derived. Second, the multi-objective optimization problem was formulated in order to design a flexible structure under a periodic load in time. A new multiobjective function was also formulated in order to obtain appropriate optimal solutions which have physical meaning. Next, the optimization algorithm was constructed based on the homogenization design method. Finally, two design examples were presented to examine the characteristics of the optimal configurations. These examples confirm that the flexible structure under a periodic load can be designed using the method presented here.

Acknowledgements The first author is supported by Toyota Central R\&D Labs., Inc. This support is appreciated.

\section{References}

Allen, H.V.; Terry, S.C.; de Bruin, D.W. 1989: Self-testable accelerometer systems. In: Micro electro mechanical systems: an investigation of micro structures, sensors, actuators, machines and robots, IEEE, pp. 113-115

Ananthasuresh, G.K.; Kota, S.; Kikuchi, N. 1994: Strategies for systematic synthesis of compliant MEMS. Proc. ASME Winter Annual Meeting (held in Chicago, IL), pp. 677-686
Bendsøe, M.P. 1995: Optimization of structural topology, shape, and material, Berlin, Heidelberg: Springer

Bendsøe, M.P; Kikuchi, N. 1988: Generating optimal topologies in structural design using a homogenization method. Comp. Meth. Appl. Mech. Engrg. 71, 197-224

Burns, R.H.; Crossley, F.R.E. 1966: Structural permutations of flexible link mechanisms. ASME Paper No. 66-MECH-5

Daneman, M.J.; Tien, N.C.; Solgaard, O.; Pisano, A.P; Lau, K.Y; Muller, R.S. 1996: Linear microvibromotor for positioning optical components. J. Microelectromechanical Systems 5, 159-165

Frecker, M.I.; Ananthasuresh, G.; Nishiwaki, S.; Kikuchi, N.; Kota, S. 1997: Topological synthesis of compliant mechanisms using multicriteria optimization. J. Mech. Des., Trans. ASME 119, 238-245

Fujii, D.; Kikuchi, N. 2000: Shape and topology optimization of space shell structure using the homogenization method. Structural Engineering and Mechanics (submitted in 1998)

Greiff, P.; Boxenhorn, B.; King, T.; Niles. L. 1991: Silicon monolithic micromechanical gyroscope. Transducers '91: Proc. 1991 IEEE Int. Conf. on Solid-State Sensors and Actuators, IEEE, pp. 966-968

Guedes, J.M.; Kikuchi, N. 1990: Preprocessing and postprocessing for materials based on the homogenization method with adaptive finite element methods. Comp. Meth. Appl. Mech. Engrg. 71, 197-224

Haber, R.B.; Jog, C.S.; Bendsøe, M.P. 1996: New approach to variable-topology shape design using a constraint on perimeter. Struct. Optim. 11, 1-12

Hanson, R.; Hiebert, K. 1981: A sparse linear programming. Sandia National Laboratories, Technical Report, SAND910297

Her, I.; Midha, A. 1987: A compliance number concept for compliant mechanisms, and type synthesis. J. Mech., Trans. Auto. Des., Trans. ASME 109, 348-355

Howe, R.T. 1994: Application of silicon micromachining to resonator fabrication. Proc. 1994 IEEE 48-th Annual Symp. on Frequency Control, pp. 2-7

Huang, N. 1971: On principle of stationary mutual complementary energy and its application to optimal structural design. J. Appl. Math. Phys., ZAMP 22, 609-620

Huang, Q.; Kuratli, C. 1996: Ultrasound source based on a micro-machined electromechanical resonator. Proc. 1996 IEEE Int. Symp. on Circuits and Systems, ISCAS. Part 4 (of 4), IEEE, pp. 348-351

Kagawa, Y.; Tsuchiya, T.; Kataoka, T. 1996: Finite element simulation of dynamic response of piezoelectric actuators. J. Sound \& Vib. 191, 519-538

Kohn, R.V.; Strang, G. 1986: Optimal design and relaxation of variational problems. Comm. Pure Appl. Math. 39, 1-25 (Part I), 139-192 (Part II), 353-377 (Part III)

Koski, J. 1985: Defectiveness of weighting method in multicriteria optimization of structures. Comm. Appl. Numer. Methods 1, 333-337

Koski, J. 1993: Multicriteria optimization in structural design: State of the art. Proc. ASME Advance in Design Automation DE-Vol. 65-1, pp. 621-629

Lee, A.P.; Pisano, A.P. 1992: Polysilicon angular microvibromotors. J. Microelectromechanical Systems 1, 70-76 
Ma, Z.-D.; Kikuchi, N.; Cheng, H.-C. 1995: Topological design for vibrating structures. Comp. Meth. Appl. Mech. Engrg. 121, 259-280

Murat, F.; Tartar, L. 1985: Optimality conditions and homogenization. In: Mairo, A.; Modica, L.; Spagnolo, S. (eds.) Nonlinear variational problems, pp. 1-8. Boston: Pitman Publishing Program

Nguyen, C.T.-C. 1996: Micromechanical resonators for oscillators and filters. Proc. 1995 IEEE Ultrasonics Symp., pp. 489-499

Nishiwaki, S.; Frecker, M.I.; Min, S.; Kikuchi, N. 1998: Topology optimization of compliant mechanisms using the homogenization method. Int. J. Numer. Meth. Eng. 42, 535-650

Onitsuka, K.; Dogan, A.; Tressler, J.F.; Xu, Q.; Yoshikawa, S.; Newnham, R.E. 1995: Metal-ceramic composite transducer, the "Moonie". J. Intel. Mat. Sys. Struct. 6, 447-455

Pedersen, P. 1989: On optimal orientation of orthotropic materials. Struct. Optim. 1, 101-106

Petersen, K.E. 1980: Silicon torsional scanning mirror. IBM J. Research Development 24, 631

Saitou. K.; Wou., S. 1998: Externally-resonated micro linear vibromotor for micro assembly. Proc. 1998 SPIE Int.
Symp. on Intelligent Systems Advanced Manufacturing (held in Boston, Massachusetts) pp. 128-139

Seemann, W. 1996: Ultrasonic traveling wave linear motor. Smart Structures and Integrated Systems, Seminar Proc. Int. Soc. Opt. Eng., SPIE 2717, 554-564

Shield, R.T.; Prager, W. 1970: Optimal structural design for given deflection. J. Appl. Math. Phys., ZAMP 21, 513-523

Sigmund, O. 1996: On the design of compliant mechanisms using topology optimization. Danish Center for Appl. Math. Mech. 535, 1-28

Stadler, W. 1988: Fundamentals of multicriteria optimization. In: Stadler, W. (ed.) Multicriteria optimization in engineering and in the sciences, pp. 1-25. New York: Plenum Press

Suzuki, K; Kikuchi, N. 1991: A homogenization method for shape and topology optimization. Comput. Methods Appl. Mech. Engrg. 93, 291-318

Thornton, K.E.B.; Uttamchandani, D.; Culshaw, B. 1988: Optically excited micromechanical resonator pressure sensor. Optical Fiber Sensors, 1988 Technical Digest Series, Vol. 2, Parts 1-2, IEEE, ii/433-436

Zhang, B.; Zhu, Z. 1997: Developing a linear piezomotor with nanometer resolution and high stiffness. IEEE/ASME Trans. on Mechatronics 2, 22-29 